\title{
Driver Drowsiness Detection System
}

\author{
Jayasenan J. S ${ }^{1}$, Mrs. Smitha P. S \\ ${ }^{I}$ Student, M.Tech Signal Processing, Department of electronics and communication engineering, Sree Chithra \\ Thirunal College of Engineering and Technology, Pappanamcode, Trivandrum \\ ${ }^{2}$ Assistant Professor, Department of electronics and communication engineering,Sree Chithra Thirunal College \\ of Engineering and Technology, Pappanamcode,Trivandrum
}

\begin{abstract}
The advancement of technologies for averting drowsiness at the wheel is a key dilemma in the field of accident prevention systems. Preventing drowsiness during driving necessitates a scheme for precisely perceiving deterioration in driver's vigilance and a means for alerting and reviving the driver. Drowsy Driver Detection System has been developed, using a non-intrusive machine vision based concepts. This system offers a method for driver eye detection, which could be used for observing a driver's fatigue level while he/she is maneuvering a vehicle. The system uses a small monochrome security camera that points directly towards the driver's face and monitors the driver's eyes in order to detect fatigue. In such a case when fatigue is detected, a warning signal is issued to alert the driver. This paper describes the method that has been proposed for finding the eyes, and also for determining if the eyes are open or closed. The system deals with using information obtained for the contour of the image to find the edges of the face, which narrows the area of where the eyes may exist. Once the face area is found, the eyes are found by computing the contour. Once the eyes are located, converting to binary and by counting the bright pixels in the eye area determine whether the eyes are open or closed. The system is also able to detect when the eyes cannot be found, and works under reasonable lighting conditions.
\end{abstract}

\section{Introduction}

Sleep-divested driving is the process of functioning a motor vehicle while being continuously messed up by lack of sleep. It is a key trigger of many motor vehicle catastrophes, and it can blight the human brain as $\mathrm{m} \mathrm{uch}$ as alcohol $\mathrm{c}$ an. Drowsiness influe nce mental alertness, intensifying the risk of can be triggered by numerous causes such as fatigue, lack of sleep and the use of medication. In addition, an altemative reason that can be $\mathrm{conte} m p l a t e d$ is the dullness of driving on expressways or in jam-packed traffic. The persistent construction of highways and upgrading of vehicle operation have made it feasible for drivers to benefit from enjoyable and rest ful driving. Conversely, drivers are more pertinent to maneuvers their vehicles under tedious driving circumstances.

The development of a scheme for distinguishing or avoiding drowsiness at the controls is a major contest in the domain of accident averting practices Probable procedures for perceiving drowsiness in drivers can be generally classified into four major classes: sensing of physiological characteristics, sensing of driver operation, sensing of vehicle response, monitoring the response of driver. Among these different methods, the best detection precision is accomplished with methods that are supported on physiological prodigies. Physiological measurements can be executed in two approaches: determining variations in physiological signals, such as brain waves, heart rate etc using electrodes; and measuring physical changes such as sagging posture, leaning of the driver"s head and the open/closed states of the eyes. An EEG-based system developed by [1] was able to detect fatigue with an error percentage of around $10 \%$, which the authors allege to be very steadfast. Related EEG centered analyses were also conducted by [2], [3] and [4]. The foremost disadvantage with these systems is that electrodes have to be appended to driver, producing frustration to him.

The vehicle restraining schemes that might be used for getting the impression of driving operation comprises the steering wheel, accelerator, brake pedal or transmission shift lever. The vehicle performance perceived might be the vehicle speed, lateral acceleration, yaw rate or lateral displacement. Since these practices permit noncontact recognition of drowsiness, they do not provide the driver any impression of uneasiness. On the damaging part, they are focused to several precincts depending on the vehicle category and driving circumstances. It would also be essential to formulate distinct detection logic for each type of vehicle. Still a different dilemma with this tactic is that detection would not be feasible at minimal pace.

An analysis of the human eyes under a provision of $\mathrm{diminished} \mathrm{vigilance} \mathrm{specifies} \mathrm{that}$ 
the eyes are constricted than in a wide-awake status and that there are occasions when the eyes actually close.

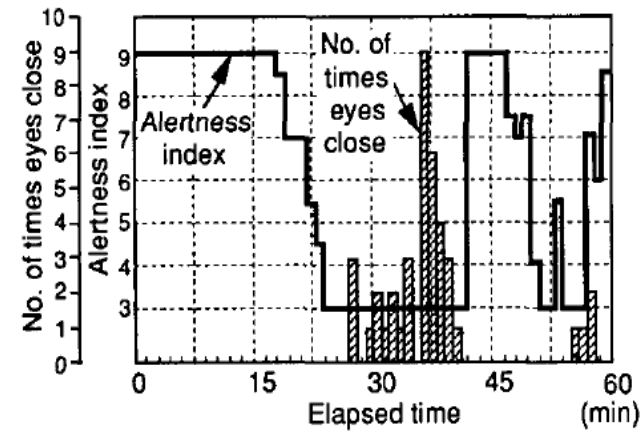

Fig 1: No of times eyes close and alertness level

Figure1 offers experimental findings demonstrating the vigilance level and the number of times the driver's eyes closed for two or more seconds while driving on a test course. Good correlation is seen between the two sets of data. This result indicates that a reduced level of alertness could be detected with good accuracy by monitoring changes in the degree of openness of the driver's eyes.

Second section explains the proposed method. In the third section $\mathrm{meth}$ o d o lo g y is explained. Results have been explained in fourth section and fifth section is conclusion and future scope

\section{Proposed Method}

In the proposed system, the optical cues of the driver, captured using a camera has been used for building on a non-invasive monitoring scheme that will not divert the driver and simultaneously which will assure precision in detecting drowsiness..Fig 2 shows the basic block diagram. A small CCD camera positioned in front of the driver behind the steering in a position will be taking continues videos of the driver's face. The camera should be positioned such that the driver"s face takes up the greater part of the image and also that the driver"s face should be more or less in the centre of the image.

The video will be transformed to frame by frame data and remitted to the frame memory of the image processor. The frame memory will be hoarding each image in a appropriate pixel format. A personal computer will be linked to the image processor for manipulating the image proce s s in procedure and forascertaining the processed results. The functions of the system can be broadly divided into face detection and eye detection function, embracing the first half of the processing schedule, and a drowsiness detection function, including the second half. Figure 2 shows the block diagram of the eye detection process.

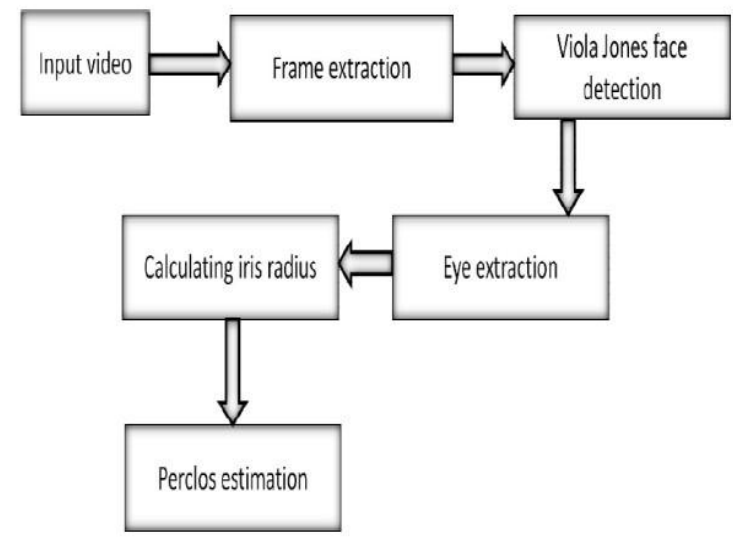

Fig 2: Block Diagram

\subsection{Flowchart of major functions}

\section{Methodology}

It has been stated that the proposed method work very well for IR images. Fig 3 shows the flowchart of major functions.

\subsection{Face Detection}

At first the video of driver is captured using an IR camera which is fixed in front of the driver behind 
the steering in a position so that driver"s face can be clearly captured. Then the input video is converted to frames and each frame is processed separately. Viola Jones method of face detection is then used. This procedure classifies images based on the value of simple features. The simple features used are reminiscent of Haar basis functions. More specifically, three kinds of features are used. The value of a two-rectangle feature is the difference between the sum of the pixels within two Rectangular regions. The regions have the same size and shape and are horizontally or vertically adjacent. A three-rectangle feature computes the sum within two outside rectangles subtracted from the sum in a centre rectangle. Finally a four-rectangle feature computes the difference between diagonal pairs of rectangles. A cascade of classifiers is then constructed to achieve increased detection performance while radically reducing computation time. The overall form of the detection process is that of a degenerate decision tree. A series of classifiers are then applied to every sub-window. The initial classifier eliminates a large number of negative examples with very little processing. Subsequent layers eliminate additional negatives but require additional computation. After several stages of processing the number of sub-windows has been reduced radically. Further processing can take any form such as additional stages of the cascade (as in detection system) or an alternative detection system. Much like a decision tree, subsequent classifiers are trained using those examples which pass through all the previous stages. The cascade design process is driven from a set of detection and performance goals.

\subsection{Eye Extraction}

The circumference of the iris is then estimated using the Hough transform for circle detection from curves. So that iris is considered as a circular object and using the principle of Hough transform, the circular iris is extracted and the visible circumference of iris is calculated. In Hough transform, the points are linked by determining first if they lie on the curve of specified shape. This method detects both straight line and circle. Circles are then drawn on the original image.

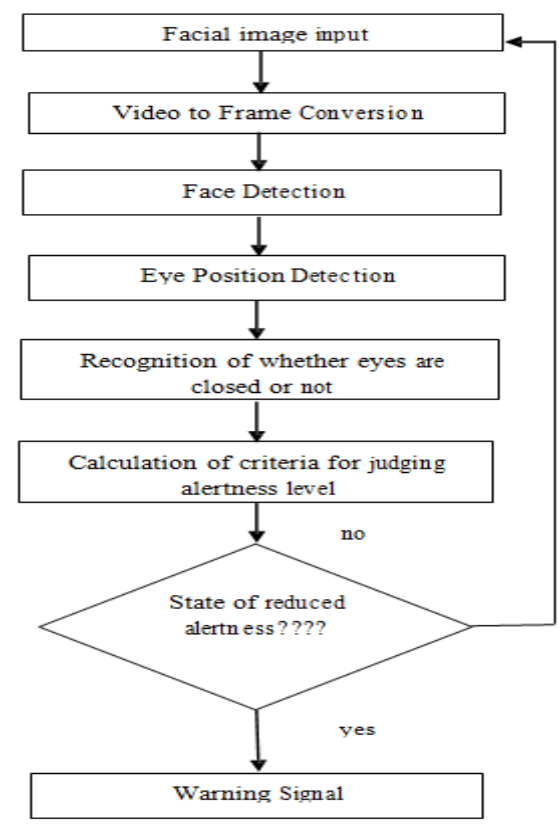

Fig 3 Flowchart of major functions

\subsection{Perclos Estimation}

The percentage of closure of eye is same as the number of pixels visible at the circumference of the iris. So the instantaneous circumference value is compared with the pervious circumference value. Once the percentage of closure is found, and if fatigue is detected a warning signal is issued to alert the driver.

\subsection{Drowsiness detection}

The alert to driver is issued based on the decision from face detection section and perclos estimation section. The alert is given when the face is not detected and when perclos value of adjacent 2 frames is less than $0.7^{*}$ maximum value , $\mathrm{c}^{* \prime}$.

\section{Results}

In this method a novel algoritm is introduced This method is effective in normal web cam videos in sufficient lighting conditions. Fig 4 shows the face and eye detected image and Fig 5 sho ws the estimated circle of the detected eye.shows the final output. 


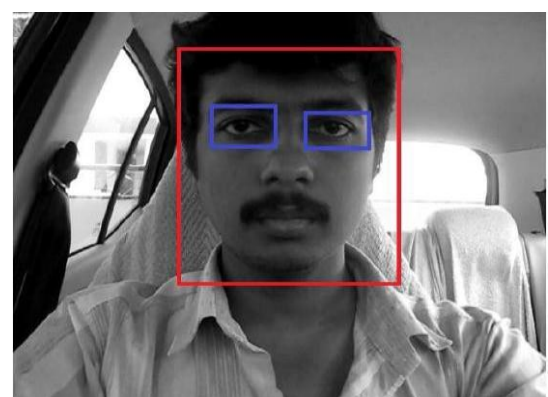

Fig 4: Face detection and eye detection
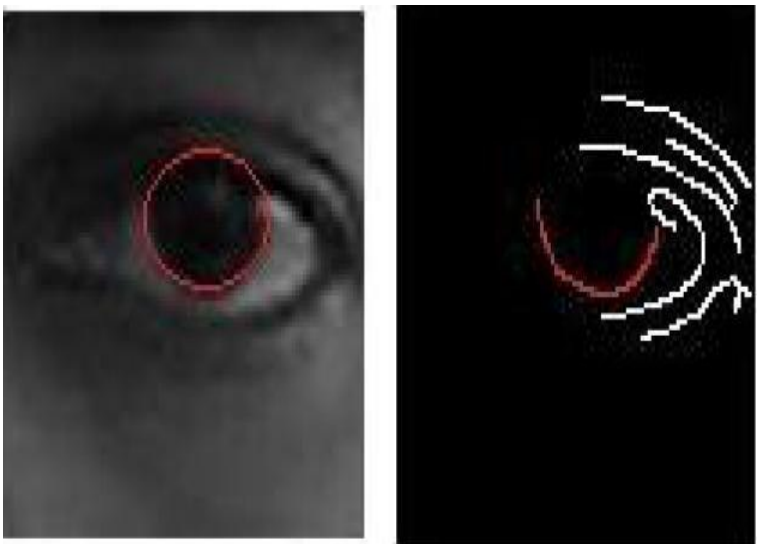

Fig 5: Detected and estimated circle of eye

\section{Conclusion and future scope}

In this paper a novel method is introduced which is simple in terms of algorithm and gives more accuracy compared to existing methods A non-insidious system to localize the eyes and monitor fatigue was developed. Information about the head and eyes position is obtained through various image processing algorithms. During the monitoring, the system is able to decide if the eyes are opened or closed. When the eyes have been closed for too long, a warning signal is issued. Image processing achieves highly precise and consistent detection of drowsiness and offers a non-insidious approach to detecting drowsiness without the infuriation and hindrance. A non-insidious system developed judges the driver"s alertness level on the basis of continuous eye closures. There are a number of issues that remain to be addressed in the system. These include improvement of its flexibility to changes in ambient brightness, assurance of consistency and attainment of a more compact system design.

\section{Reference}

[1] S. G. Klauer, T. A. Dingus, V. L. Neale, and J. D. Sudweeks, "The impact of driver inattention on near-crash/crash risk: An analysis using the 100-car naturalistic driving study data," National Highway Traffic Safety Administration, DC, DOT HS, vol. $810,2006$.

[2] T. Akerstedt, G. Kecklund, and L. H"orte, "Night driving, season, and the risk of highway accidents." Slee, vol. 24, pp. 401406, 2001.

[3] J. Connor, R. Norton, S. Ameratunga, E. Robinson, I. Civil, R. Dunn, J. Bailey, and R. Jackson, "Driver sleepiness and risk of serious injury to car occupants: Population based control study." British Medical Journal, vol. 324, pp. 1125- 1129, 2002.

[4] J. Horne and L. Reyner, "Vehicle accidents related to sleep: A review." Occupational and Environmental Medicine, vol. 56, pp. 189-294, 1999.

[5] L. M. Bergasa, J. Nuevo, M. A'. Sotelo, R. Barea, and M. E. L. Guille'n, "Realtime system for monitoring driver vigilance," IEEE Transactions on Intelligent Transportation Systems, vol. 7, no. 1, pp. 63-77, 2006.

[6] C. Papadelis, Z. Chen, C. Kourtidou-Papadeli, P. Bamidis, I. Chouvarda, E. Bekiaris, and N. Maglaveras, "Monitoring sleepiness with on-board electrophysiological recordings for preventing sleep-deprived traffic accidents." Clinical Neurophysiology, vol. 118, no. 9, pp. 1906-1922, September 2007.

[7] J. Faber, "Detection of different levels of vigilance by eeg pseudo spectra." Neural Network World, vol. 14, no. 3-4, pp. 285290, 2004.

[8] N. Wright, B. Stone, T. Horberry, and N. Reed, “ A review of in-vehicle sleepiness detection devices," TRL Limited, Published Project Report PPR157, 2007.

[9] T. Wakita, K. Ozawa, C. Miyajima, K. Igarashi, K. Itou, K. Takeda, and F. Itakura, "Driver identification using driving behavior signals," IEICE - Trans. Inf. Syst., vol. E89-D, no. 3, pp. 1188-1194, 2006.

[10] Y. Takei and Y. Furukawa, "Estimate of driver"s fatigue through steering motion." IEEE International Conference on Systems, Man and Cybernetics., vol. 2, p. 1765-1770, 2005. 